\title{
An experimental study of the electromotive forces of the heart
}

\author{
Ernest W. Reynolds, Jr., M.D.* \\ Daniel A. Weller, M.D.** \\ Ann Arbor, Mich.
}

$\mathrm{P}$ rogress in understanding the relationship between differences in potential measured in a lead and the electromotive forces of the heart has been closely related to the improved understanding of the nature of these forces and the development of the concepts of the lead vector ${ }^{1}$ and lead field. ${ }^{2}$ The latter concepts permit us to explain how the electromotive forces of the heart produce voltage in leads in finite and nonhomogeneous media. With regard to the electromotive forces themselves, the studies of Craib and Canfield ${ }^{3}$ and Wilson ${ }^{4}$ replaced the crude bipole model proposed by Waller, ${ }^{5}$ in 1889 , with a more sophisticated picture of the heart's electrical activity as generated by a complex of electromotive surfaces located at the junction between active and resting muscle. At any instant of time this junction constitutes a continuous layer within the confines of the heart, with its positive side toward resting tissue and its negative side toward active tissue. A single element of this surface produces an electrical field equivalent to that produced by a dipole consisting of a point source and point sink of current, of equal size and close to one another.

The potential equation for an electromotive surface consisting of two layers placed in a resistive, linear, and isotropic media of infinite size is:

$$
V= \pm \frac{1}{4 \pi} \int_{s} E \mathrm{~d} \omega+C
$$

where $\mathrm{V}$ is the potential at a point in the media, $\mathrm{E}$ is the difference in potential across a single element of the electromotive surface, and $d \omega$ is the solid angle subtending a single element of the electromotive surface. The constant, $C$, used when evaluating a single potential disappears when cvaluating the difference in potential between two points in the media. If $\mathrm{E}$ is constant for every element of electromotive surface, then integration yields:

$$
\mathrm{V}= \pm \frac{\mathrm{E}}{4 \pi} \cdot \Omega+\mathrm{C}
$$

where $\Omega$ is the solid angle subtending the boundary of the entire electromotive surface. The fact that the surrounding medium is nonhomogeneous and finite does not substantially detract from the usefulness of this equation, since it is usually approximately true even for finite nonhomogeneous conducting media.

In the special case of a closed electromotive surface of any shape, the difference in potential between any point inside the closed surface and any outside point is

\footnotetext{
This study was supported in part by Public Health Service Research Grant HE 07406-02 from the National Institutes of Health, and by the Maximillian Reba E. Richter Fund.

Received for publication March 9, 1964.

*Associate Professor of Internal Mediciste, Universicy of Michigan Medical Center, Anu Arbur, Mich.

* Senior Medical Student, University of Micligan Medical School, Ann Arbor, Mich.
} 
simply equal to the difference in potential, $E$, across the electromotive surface. The difference in potential between any two points outside a closed electromotive surface is zero, provided that the difference in potential across the electromotive surface is uniform. In the special case under consideration these conclusions are applicable even in a nonhomogeneous conducting media.

The relations developed for an electromotive surface apply at a single instant of time for a propagated wave; thus, the situation must be analyzed from instant to instant, treating each position of the wave as static. The dimensions of a real wavefront are finite. Durrer's ${ }^{6}$ measurements indicate that the wavefront in the outer three fifths of the myocardial wall is sharply bounded and regular in form. The distance between the sources and sinks of the activation wave in these layers is ordinarily less than $1 \mathrm{~mm}$.

The present study is an attempt to measure the external field associated with propagated activity which should, in theory, produce a closed electromotive surface. The value of this type of experiment is that it provides evidence that a propagated wavefront has a uniform difference in potential across its surface.

\section{Methods}

Ten dogs were anesthetized with pentobarbital, $50 \mathrm{mg}$. per kilogram, intubated, and maintained with an alternator typc of respirator. The chest was opened long enough to permit implanting the stimulating and recording electrodes. Since one requirement of the study was to permit simultaneous stimulation and recording from the same intramural site, ordinary electrical methods of stimulation which tend to block the recording amplifiers cannot be used. To remedy this, mechanical stimulation was used, since this method produces less stimulation artifact. Early in this study a simple wire probe placed inside a hypodermic needle which was insulated except at the tip was used to mechanically stimulate the heart at a known depth. ${ }^{7}$ The wire probe was simply tapped by hand until by chance the mechanical stimulus so produced fell after the refractory period and produced a propagated beat. To obviate the difficulties caused by the refractory period of the heart, a method of synchronizing the mechanical stimulus to the cardiac electrical cycle was developed. This was accomplished by driving the atria at a constant rate with an electronic stimulator. A second stimulator synchronized to the first by a variable delay drove a relay, which, in turn, actuated a solenoid to deliver the mechanical stimulus. The solenoid, shown in Fig. 1, contained 25 turns of No. 22 copper wire wound around an iron core. On the application of a 20-Insec., 6-volt D.C. pulse from the relay, the self-contained stylusplunger descended sharply $1 \mathrm{~mm}$. beyond the tip of the hypodermic needle affixed to the base of the solenoid. In actual use the needle was inserted to a depth of from 2.5 to $6 \mathrm{~mm}$. into the myocardium, and the solenoid was sewn to the surface of the heart. This arrangement permitted the automatic delivery of the mechanical stimulus at any time in the cardiac cycle desired. The base of the solenoid and the tip of the needle acted as recording electrodes. In addition, other plunge elec-

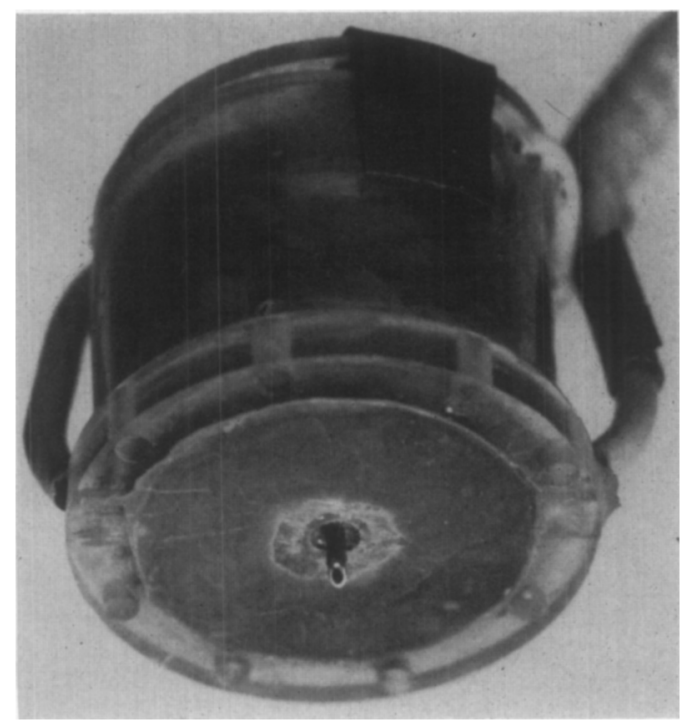

Fig. 1. The solenoid stimulator for producing a mechanically stimulated intramural ectopic beat (closed electromotive surface). The plunger that produces mechanical stimulation is contained within the hypodermic needle. The tip of the needle serves as central (intramural) recording electrode, and the round silver disk mounted flush with the plastic base is the surface recording electrode. 
trodes were placed near the endocardial surface and at various depths within the myocardium, lateral to but within a radius of approximately $10 \mathrm{~mm}$. from the tip of the stimulator. After the electrodes had been sewn in place, the dog's chest cage was closed.

To record accurately the small time differences involved, the electrograms were recorded on a Hathaway oscillograph having a paper speed of 1,130 or $620 \mathrm{~mm}$. per second. The mirror type of coil galvanometers used were driven by Tektronix Type 122-S1 preamplifiers, so that the over-all frequency response was 0.04 to 210 c.p.s. The voltage was reduced 36 per cent at the latter frequency.

Twenty-four experiments were carried out in 3 dogs, using the hand-operated wire-probe stimulator, and 80 experiments were carried out in 7 dogs, using the solenoid. The results were similar with both methods of mechanical stimulation, although fewer artifacts were seen with the hand-operated stimulator. Synchronous recordings were made from the tip of the needle, called the central lead, and one or more of the electrodes, placed around the central lead point, usually the adjacent ventricular surface, and occasionally from one of the electrodes located on the endocardium or at the same depth in the myocardium but lateral to the central lead point. No special advantage is attributed to one of these recording sites, and the surface electrode was the simplest to use, since the distance between the central tip and the surface electrodes was fixed and could be measured accurately. The right leg was used as the reference voltage.

\section{Results}

The central lead showed an initial negative deflection in all 24 of the hand-operated mechanical stimulator experiments, and in 58 of 80 solenoid experiments. In the latter, unpredictable artifacts were produced in the central lead, and in 26 of these a small initial positive deflection preceded the main negative deflection. Since the intervals between the onset of electrical activity in the central lead and nearby surface or intramural sites were similar in both sets of measurements, and the isoelectric interval in the surface lead was not disfigured by these artifacts, the results obtained using the solenoid technique were retained. Electrical activity as measured by any displacement of the base line as recorded at the central electrode

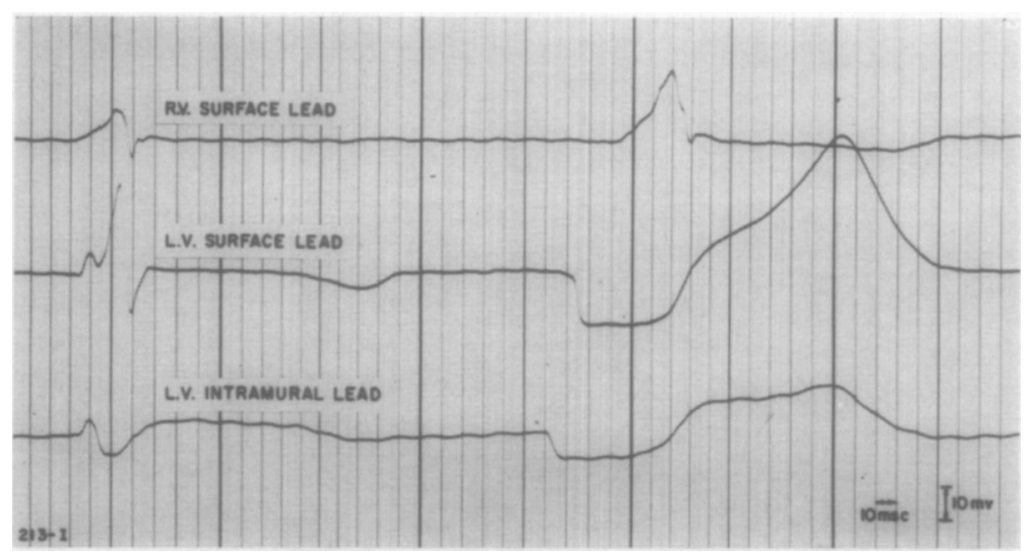

Fig. 2. The normally propagated beat seen at the left shows synchronous onset of electrical activity, but in the intramural ectopic beat at the right there is a $12.1-\mathrm{msec}$. period in which the left ventricular surface lead is isoelectric with respect to the intramural lead located inside the closed double layer. The electromotive surface penetrated the epicardial surface first in this instance, since the left ventricular surface lead is negative. The upper trace is part of a study of open electromotive surfaces and shows that a lead over the right ventricle is insensitive to the early changes over the left ventricle because of the great distance involved and the small size of the electromotive surface at this time. 
preceded that at the adjacent surface recording by a mean value of $10.6 \mathrm{msec}$. in the hand-operated stimulator experiments where the stimulator tip was placed at a depth of $5 \mathrm{~mm}$. in the myocardium. Thus, the mean conduction velocity for the $5-\mathrm{mm}$. distance separating the two recording electrodes was $470 \mathrm{~mm}$. per second in this group of experiments. Fig. 2 is an example of the propagated wave reaching the epicardial surface first as indicated by a negative deflection in the left ventricular surface lead. The period between the onset of electrical activity at the central lead and the onset of electrical activity at the left ventricular surface lead is 12.1 msec., and during this interval the difference in potential produced in the left ventricular surface lead is negligible. Recordings made from these electrodes during the production of extrasystolic beats at many other sites or during normal activity showed electrical activity which was considered to be synchronous; i.e., the onset of the deflection in the several leads was within 1 or $2 \mathrm{msec}$. as compared with the 5 to $23 \mathrm{msec}$. for the intramural beat described. An example of a normally propagated beat is shown on the left-hand side of Fig. 2.

In one instance illustrated in Fig. 3, propagation reached the endocardial surface first, as shown by the positive deflection in the left ventricular surface lead. The isoelectric interval between the onset of electrical activity at the central electrode and the left ventricular surface lead was $23.2 \mathrm{msec}$. This interval is almost twice as long as that observed in the other experiments and suggests that propagation to the endocardium pursued a tangential course. In this example, as in all of the others, the external field produced by the propagated wave is negligible until the wave reaches the ventricular surface. Table I gives a summary of all of the measurements made over the anterior and posterior walls of the left ventricle and the anterior wall of the right ventricle. The column on the right which gives the calculated depth of stimulation serves as a check on the positioning of the electrode tip. The measurement $I$ to $S$ is the duration of the isoelectric interval. The variations in voltage during this interval were of the same order of magnitude as shown in

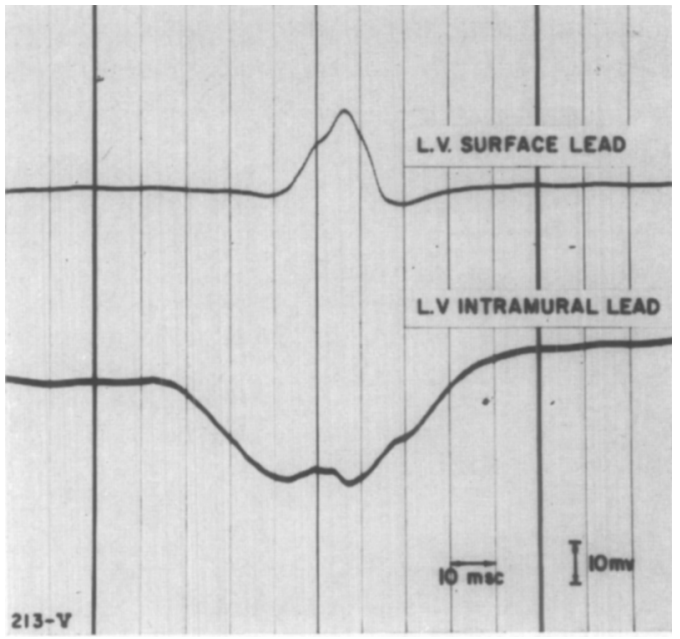

Fig. 3. Synchronous recordings with exploring electrodes located $5 \mathrm{~mm}$. apart and using the right leg as a common reference. The intramural electrode located at the point of origin of the propagated beat records an initial negative voltage, whereas the surface lead remains isoelectric for $23.2 \mathrm{msec}$. Activation must have penetrated the endocardial surface first, since the surface lead is positive, and the solid angle subtended at the surface point by an open electromotive surface would then face the positive side of the open electromotive surface.

Figs. 2 and 3 . The sensitivity of the recordings was $10 \mathrm{mv}$. $=1 \mathrm{~cm}$., which is considered to be adequate for direct leads.

\section{Discussion}

It is assumed in this study that propagated wavefronts which do not present to either the epicardial or endocardial surfaces are temporarily closed electromotive surfaces. Since the external field produced by these closed electromotive surfaces is negligible, even when measured by external electrodes placed a few millimeters distance from the wavefront, this strongly suggests that the difference in potential across these closed electromotive surfaces is uniform, or otherwise such self-cancellation would not occur. Certainly, the small variations in potential observed during the isoelectric interval as depicted in Figs. 2 and 3 would not be measurable at the body surface.

It is reasonable to assume that propagated wavefronts have similar characteristics whether they are closed or whether they have boundaries on the epicardial or endocardial surfaces of the heart. There- 
Table I. Summary of measurements of the duration of the isoelectric period from the onset of electrical activity at the central stimulating electrode (I) to the superjacenl surface electrode $(S)$

\begin{tabular}{|c|c|c|c|}
\hline $\begin{array}{l}\text { Depth of electrode } \\
\text { tip (mm.) }\end{array}$ & Number of measurements & $\begin{array}{l}\text { Mean duration } \\
\text { I to } S(\text { msec. })\end{array}$ & $\begin{array}{l}\text { Calculated depth of } \\
\text { stimulation }{ }^{*}(\mathrm{~mm} .)\end{array}$ \\
\hline \multicolumn{4}{|c|}{ Anterior wall of left ventricle } \\
\hline 4 & 24 Solenoid & 8.5 & 4.2 \\
\hline 5 & 24 Hand stimulated & 10.6 & 5.3 \\
\hline \multicolumn{4}{|c|}{ Posterior wall of left ventricle } \\
\hline 2.5 & 27 Solenoid & 7.4 & 3.0 \\
\hline 6 & 6 Solenoid & 8.3 & 4.1 \\
\hline \multicolumn{4}{|c|}{ Anterior wall of right ventricle } \\
\hline 2.5 & 16 Solenoid & 7.5 & 3.7 \\
\hline 5 & 7 Solenoid & 8.4 & 4.2 \\
\hline
\end{tabular}

*Assuming linear propagation in the outer layers of $500 \mathrm{~mm} . / \mathrm{sec} . \times($ I-S interval $)=$ calculated depth.

fore, the present studies would also suggest that a uniform difference in potential exists across all propagated wavefronts in normal ventricular muscle.

Although the absolute value of $E$, the difference in potential across the electromotive surface, could not be measured accurately in the present study, it is obvious from inspection of Fig. 3 that $E$ has a value of the order of one sixth to one fourth of the transmembrane resting potential to which it is related. This reduction in voltage is believed to be related to the physical separation of cardiac fibers by interstitial spaces containing capillaries and producing holes in the elcctromotive surface, as well as the shunting effects of interstitial fluids, and by other factors as discussed by Scher. ${ }^{8}$ If these holes were uniformly distributed, the closed electromotive surface would still be self-cancelling, but would show a reduced voltage across its surface.

Some consideration should be given to the possibility that the data as derived from this study are subject to other interpretations. The mass of tissue involved in the spread of activation which follows intramural stimulation may be too small to give a recognizable deflection in the adjacent ventricular surface lead, whether the electromotive surface is uniform or not. The other possibility is that the observed voltage cancellation which occurs in surface leads is the result of a peculiar distribution of electromotive forces across the electromotive surface which may cause cancellation, but which is far from uni- form. One may visualize a quadripolar distribution of electromotive forces where the external field would not be zero but would be very small.

It is thought that these objections are not serious, since the variations in potential observed in leads located as close as $2.5 \mathrm{~mm}$. to the centrally stimulated point were no greater than those observed in more distant leads. The possibility of cancellation occurring as a result of an unusual distribution of electromotive forces on the electromotive surface is thought to be small, since the large number of measurements of the right, left lateral, and diaphragmatic portions of the heart should exclude this from occurring by chance. In addition, other measurements using differential electrodes point to the fact that the spread of activation during these experiments was not concentric or symmetrical, but generally followed the direction of cardiac fibers. Since the closed electromotive surfaces produced were not of symmetrical shape, it is difficult to believe that the observed cancellation could be produced by any but a uniform distribution of electromotive forces.

Examples of closed electromotive surfaces in heart muscle. The resemblance between the cardiac resting cell and a closed electromotive surface is well known. In this instance the cell has a uniform difference in potential of $90 \mathrm{mv}$. across its membrane, and no difference in potential is measurable outside the resting cell, even when electrodes are adjacent to the cell. Durrer ${ }^{6}$ 
postulates that the inner layer of the myocardium is laced with rapidly conducting Purkinje fibers which give rise to an activation wave in the epicardial direction and also to the endocardial direction. If this is the case, such activity would be an example of a closed electromotive surface, and thus would not be measurable by any combination of externally applied leads. Another example would be extrasystoles which arise wholly within the myocardium. Initially, they would not have boundaries on the epicardial or endocardial surfaces, and until propagated to the ventricular surface would not be recorded in external leads.

\section{Summary}

Propagated wavefronts were produced by mechanical stimulation at points located deeply within the myocardium, and the external field so produced was measured by electrodes located within a radius of $10 \mathrm{~mm}$. from the centrally stimulated point. In 104 experiments in 10 dogs the external field so produced was negligible until propagation reached either the epicardial or endocardial surfaces. If it is assumed that propagated intramural activity is temporarily a closed electromotive surface, then the data presented suggest that there is a uniform difference in potential across this surface. Since there is no fundamental difference in the wavefront characteristics of closed electromotive surfaces and those which present boundaries on the epicardial or endocardial surfaces, these data strongly suggest that there is a uniform difference in potential across all propagated wavefronts in normal ventricular muscle.
We wish to express our gratitude for the help of Richard McFce, Ph.D., who critically revicwed an earlier manuscript, and to Franklin D. Johnston, M.D., for much encouragement while the work was being carricd out, and for help in preparing the manuscript. We also wish to thank Robert H. Bayley, M.D., who taught the principles of an electromotive surface to us many years ago, and Vincent J. Turcotte, Jr., M.D., for help in carrying out several of the experiments reported here.

\section{REFERENCES}

1. Burger, H. C., and van Milaan, J. B.: Heart vcctor and leads, Brit. Heart J. 8:157, 1946; Part II, 9:154, 1947; Part III, Geometrical representation, 10:229, 1948.

2. McFec, R., and Johnston, F. D.: Electrocardiographic leads. I. Introduction, Circulation $8: 555,1953$.

2a. McFec, R., and Johnston, F. D.: Electrocardiographic leads. II. Analysis, Circulation 9:255, 1954.

2b. McFee, R., and Johnston, F. D.: Electrocardiographic leads. III. Snythesis, Circulation 9:868, 1954.

3. Craib, W. H., and Canfield, R.: A study of the electrical field surrounding active heart muscle, Heart 14:71, 1927.

4. Wilson, F. W., Macleod, A. G., and Barker, P. S.: The distribution of the currents of action and of injury displayed by heart muscle and other excitable tissues, University of Michigan Scientific Series, Vol. 10, Ann Arbor, 1933, University of Michigan Press.

5. Waller, A. D.: On the electromotive changes connected with the beat of the mammalian heart and of the human heart in particular, Phil. Trans. Royal Soc. London B180:169, 1889.

6. Durrer, D., van der Tweel, L. H., and Blickman, J. R.: Spread of activation in the left ventricular wall of the dog. III. Transmural and intramural analysis, Am. Heart J. 48:13, 1954.

7. Reynolds, E. W., Jr., Weller, D. A., Turcotte, V. J., Jr., and Johnston, F. D.: An experimental study of factors affecting electrocardiographic voltage, J. Lab. \& Clin. Med. 60:1010, 1962.

8. Scher, A. M., and Young, A. C.: Ventricular depolarization and the genesis of QRS, Ann. New York Acad. Sc. 65:768, 1957. 